\title{
A CPSO Algorithm for Optimization of Wireless Sensor Network Positioning
}

\author{
Xing Jin \\ Rizhao Polytechnic, Rizhao, China

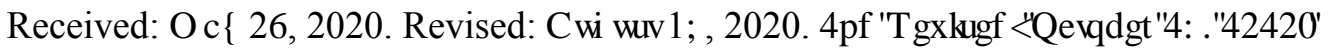 \\ Accepted: 1 RYP EHU2, 2020. [Published: November \, 2020.
}

\begin{abstract}
Wireless sensor network technology is widely used, and most applications depend on node location. Aiming at the problem that the signal intensity indication (RSSI) is susceptible to the environment, an improved chaotic particle swarm optimization (CPSO) is proposed in this paper in order to improve the positioning performance of the sensor node based on the research of the existing location algorithm. The convergence of the algorithm is better than the PSO algorithm. The results of the RSSI measurement data in both indoor and outdoor communication environments show that, compared with the general weighting algorithm and the traditional PSO algorithm, the improved combined optimization algorithm can greatly improve the effect of the ranging error on the positioning error and improve the positioning performance greatly.
\end{abstract}

Keywords-wireless sensor network(WSN), localization, measurement error, particle swarm optimization.

\section{INTRODUCTION}

$\mathrm{T}$ HE Wireless sensor network is composed of a large number of discrete sensor nodes randomly deployed in the target area, and the node transmits the collected information to the base station in the form of multi-hop, which realizes the exchange of information between the physical world and the human society[1-2]. In wireless sensor networks, the information is monitored by sensor nodes, such as pressure, temperature, humidity, etc. If there is no corresponding location information without research value, the wireless sensor network to obtain the monitoring information must be accompanied by the corresponding location information in most cases, otherwise these monitoring information will lose significance. The key content of wireless sensor network research is node location technology, which is the support technology of wireless sensor network. There are several applications related to location knowledge in WSNs, such as target tracking, person tracking, monitoring, unmanned aerial vehicles, patient fall detection, wild forest areas, agriculture, disasters, and environment management [3-8].

The technology of improving the positioning accuracy is the hotspot of the wireless sensor network at present[9]. There are many kinds of localization methods for wireless sensor networks, and there are three kinds of classification methods at present.
(1) Distance-dependent algorithm and distance-independent algorithm. Distance-dependent algorithm means that the distance between nodes is used in the algorithm. On the contrary, it is called the distance independent algorithm. The positioning accuracy of distance-dependent algorithm is higher than the distance independent method, but it requires higher hardware. Distance-independent method is needed to estimate the distance between nodes when the distance is difficult to measure.

(2) Single hop algorithm and Multi-hop algorithm. The single hop algorithm is small in scope, but it is easy to realize. Multi-hop algorithm is needed in some cases with wide measurement range.

(3) Centralized algorithm and distributed algorithm. The algorithm has a high precision for monitoring and control of the application scenario, but need to consume a large amount of traffic. Due to the different data processing methods, distributed algorithm can reduce network traffic, but it is limited by node energy, calculation and storage ability.

Due to the different data processing methods, distributed algorithm can reduce network traffic, but it is limited by node energy, calculation and storage ability. Node location is the main support technology of WSN, it is one of the most basic functions of sensor network to determine the node location of acquiring message, which plays a key role in the effectiveness of sensor network application. Due to the limited energy of nodes, poor reliability, such factors as large scale and random distribution, limited communication distance of wireless module, and interference of external factors, such as wall and human body moving, have high requirements on location algorithm and positioning technology of wireless sensor network, so improving node location result has become an important topic in wireless network research[10-12]. With the development of wireless sensor network location technology, many new positioning algorithms are proposed.

Chrissavarese and so on, this paper puts forward a two-phasepositioning cyclic algorithm, first of all the rough coordinates of the nodes, then the loop refinement, can improve the accuracy, but cannot eliminate the RSSI ranging error on the location calculation effect [13]. The particle filter method is improved by using the dynamic cluster structure network, the parallel particle filter method and the information particle filter method are used for distributed estimation, which can save the 
communication energy consumption, but frequent resampling removes the particles with less weight value, resulting in the degradation of particles and the effect of precision [14-15]. At last, the coordinates of unknown nodes are computed by using three-edge measurement method or maximum likelihood estimation method. Artificial intelligence localization techniques have been used in previous research, including Artificial Neural Network (ANN), Neural Fuzzy Inference System (ANFIS), Fuzzy logic, and optimization algorithms, such as Genetic Algorithms, Particle Swarm Optimization (PSO), Bacterial Foraging Algorithm (BFA), and Gravitational Search Algorithm (GSA) [16-17].

It utilizes a population of particles to represent candidate solutions in asearch space, and optimizes the problem by iteration to move these particles to the best solutions with regard to a given measure of quality[18-20]. In the paper [21-23], the particle swarm optimization algorithm is applied to the node location, and the position of the node is better than that by maximum likelihood estimation and least square method, and the positioning accuracy is improved. However, because of the lack of global optimization ability of PSO algorithm, it is easy to fall into local optimum, which will result in large positioning error. Therefore, the particle swarm optimization algorithm must be improved to improve the positioning accuracy of wireless sensor networks.

In order to improve the positioning accuracy of particle swarm localization algorithm, this paper proposes a quantum particle swarm optimization algorithm to improve the particle swarm algorithm's speed updating formula and objective function, and to reduce the effect of ranging error on positioning accuracy. The rest of the paper is organized as follows. The PSO and improved CPSO are algorithms introduced in Section 2. Section 3 compares the performance of CPSO-based localization algorithms. Section 4 concludes the paper and presents the future work.

\section{IMPROVED CPSO ALGORITHM}

\section{A. Fundamentals of PSO Algorithm}

PSO is an algorithm, which is clustering intelligence optimization algorithm. Each particle of populations represents a feasible solution in production scheduling. Particle in the search space flies at a certain speed. It adjusts the speed according to its own "memory" and the current state of the optimal particle. Through mutual cooperation and competition between the groups, ultimately the optimal solution should be searched.

Each particle of PSO algorithm has a position vector

$$
X_{i}=\left(x_{i, 1}, x_{i, 2}, \cdots, x_{i, n}\right)
$$

and velocity vector

$$
V_{i}=\left(v_{i, 1}, v_{i, 2}, \cdots, v_{i, n}\right)
$$

in which $i=1,2, \cdots, p$ size, psize is the population size, $n$ is the dimension of the search space. Each particle has its own best position vector

$$
\text { pbest }_{i}=\left(p_{i, 1}, p_{i, 2}, \cdots, p_{i, n}\right)
$$

And the global optimal position vector of the entire population is.

$$
p_{g}=\left(p_{g, 1}, p_{g, 2}, \cdots, p_{g, n}\right)
$$

Update formulas of particles' velocity vector and position vector are as follows:

$$
\begin{gathered}
v_{i, j}^{k+1}=w \cdot v_{i, j}{ }^{k}+c_{1} \cdot r_{1} \cdot\left(p_{i, j}^{k}-x_{i, j}^{k}\right) \\
+c_{2} \cdot r_{2} \cdot\left(p_{g, j}^{k}-x_{i, j}^{k}\right) \\
x_{i, j}^{k+1}=v_{i, j}^{k}+x_{i, j}^{k}
\end{gathered}
$$

Where $k$ is the current iteration number, $w$ is the inertia weight factor, $c_{1}$ and $c_{2}$ are the positive constant acceleration factor, $r_{1}$ and $r_{2}$ are random numbers which uniformly distributed between $0-1$. Each dimension of particle's position vector and velocity vector has upper and lower bounds, they can be set separately as $\left[x_{\min }, x_{\max }\right]$ and $\left[v_{\min }, v_{\max }\right]$.

\section{B. Improved Chaos Operator}

Standard PSO algorithm can solve the scheduling problems of fewer types of products. When the product species are very large, the number of product scheduling order grows exponentially. Standard PSO algorithm easily falls into local optimum. Introducing chaotic maps can improve population particle capacity to escape from local optimum and reduce the number of iterations computing.

Chaotic maps can generate pseudo-random, iterative traversal solution.It is suitable for updating the position vector of the particle populations. Chaotic map for updating the particle position vector is required to have a uniform distribution, while reducing the steady-state solution or periodic points. Logistic chaotic mapping is a widely used mapping, it has no stable periodic points or steady-state solution in the whole interval $[0,1]$. However, it's iterative point in the interval $[0,1]$ being the Chebyshev distribution. In contrast, Tent Mapping is a one-dimensional piecewise linear map[24]. It has uniform probability density, the probability density in the interval $[0,1]$ is 1 . But Tent Mapping in $2 / 3$ point has steady-state solution, and there are little unstable periodic points.

In response to these two common chaotic mapping's own characteristics, this paper introduces a Tent mapping with superimposed Logistic perturbation. It eliminates the steady-state solution of Tent by small perturbations. At the same time it almost does not affect the magnitude of the iteration points, thus improving chaos operator still maintained a good uniform distribution. The formula is as follows : 


$$
x^{t+1}=f\left(x^{t}\right)=\left\{\begin{array}{lr}
2 \llbracket x^{t}+0.01 \square\left(4 \llbracket x^{t} \square\left(1-x^{t}\right)\right) & 0<x^{t} \leq 1 / 2 \\
2 \llbracket\left(1-x^{t}\right)+0.01 \square\left(4 \square x^{t} \square\left(1-x^{t}\right)\right) & 1 / 2<x^{t} \leq 1 \\
x^{t}-1 & x^{t}>1
\end{array}\right.
$$

Figure 1 is a $(0,1)$ distribution image which shows an improved model of Chaos, when the value of $x^{0}$ is 0.1 and its numbers of iteration are 500 times. The horizontal axis represents the number of iterations of chaos operator. Ordinate indicates the value of chaos iteration points. After 500 iterations, the value of chaos points can cover each corner of the interval $(0,1)$, which reflects the good performance of evenly distributing.

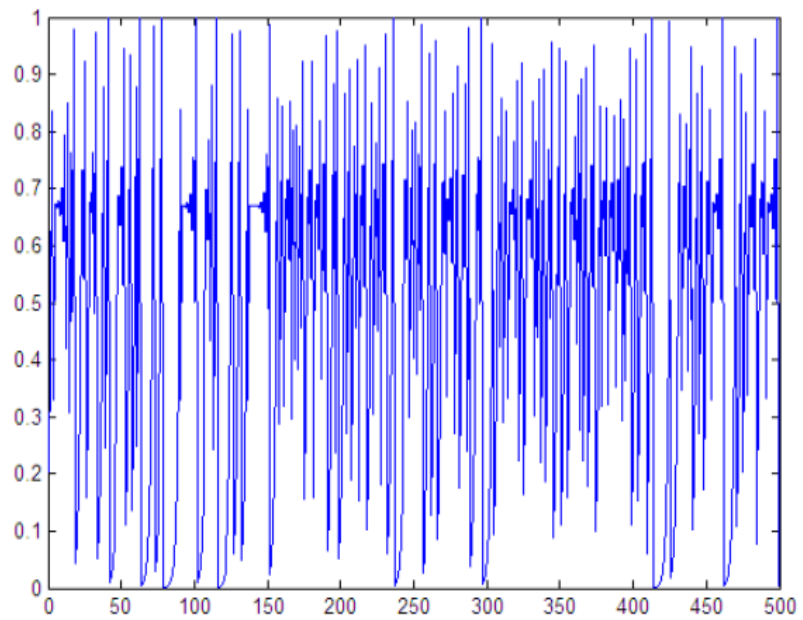

Fig.1 Iteration distribution of improved chaotic operator

According to the improved chaos operator, let $t=0$, each dimension $x_{i k}^{t}, k=1, \cdots, n$ of particles position vector $X_{i}^{t}$ should be normalized. According to equation (6), it can be mapped to the interval $(0,1)$.

$$
\left[x_{i k}^{t}\right]^{*}=\frac{x_{i k}^{t}-x_{\min , i k}}{x_{\max , i k}-x_{\min , i k}}
$$

In the above formula, $x_{\max }, i k$ and $x_{\min , i k}$ separately represents the searching upper and lower bounds of the $\mathrm{k}$-dimensional. According to the formula (5), iteration produces a chaotic point $\left[x_{i k}^{t+1}\right]^{*}$. Then in accordance with equation (7), the chaotic points will be mapped back to the original space:

$$
x_{i k}^{t+1}=x_{\min , i k}+\left[x_{i k}^{t+1}\right]^{*} \cdot\left(x_{\max , i k}-x_{\min , i k}\right)
$$

According to this, $X_{i}^{t}$ will become chaos dot columns $X_{i}^{t+1}=\left(x_{i 1}^{t+1}, x_{i 2}^{t+1}, \cdots, x_{i n}^{t+1}\right)$, after a chaotic map. If the new solution $X_{i}^{t+1}$ is better than $X_{i}^{t}$, the new solution will be output as a result of the chaotic search. Otherwise, let $t=t+1$, and continues chaos iteration.

\section{Flow of improved CPSO algorithm}

The front improved chaotic operator applied to particle swarm algorithm. The flowchart of improved CPSO algorithm is shown in Figure2.

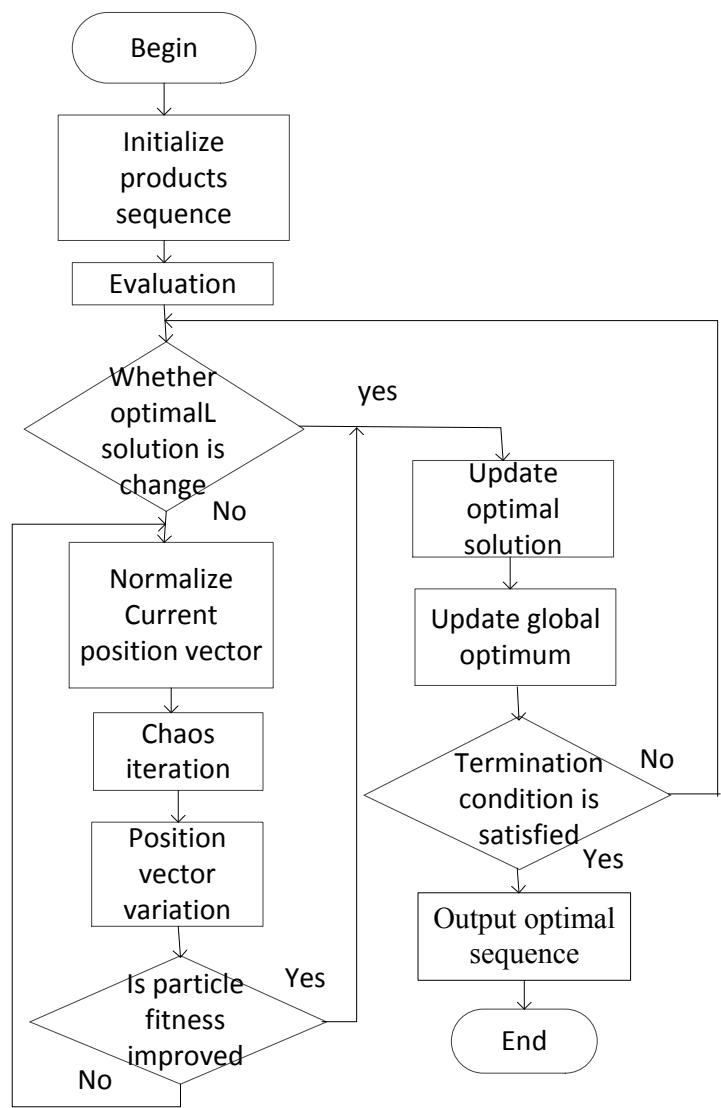

Fig.2 Flow chart of improved CPSO algorithm

For the actual optimization process as shown in Figure 2, they can be divided into the following steps.

Step 1: Initialize sequence, set the acceleration constant $c_{1}$ and $c_{2}$, weighting factor $w$, the maximum number of iterations $\mathrm{T}$, the maximum stagnancy $\mathrm{S}$. Setting the population size psize and categories $n$, then randomly generates psize position vectors and velocity vectors.

Step 2: Evaluate particle fitness, and calculate the total completion time of each node.

Step 3: According to the formula (3) and formula (4), iteration will be started. And according to the current state of the population, update the optimal solution pbest ${ }_{i}$ for each individual particle, which they experienced and global optimal solution $p_{g}$ of all particles, which they experienced.

Step 4: If they have iterated $\mathrm{S}$ times, the best fitness function value is not improved, then enter the chaos search mechanism.

Step 5: Normalized the current position vector of particles, use improved chaotic operator to iterate, calculate the next generation of chaotic sequence, through the inverse transform mapping back to the original space, then calculate the value of 
fitness function, if the value of fitness function is less than the value of current global optimum, replace a random population of particles with the position vector, and update the particles' pbest $_{i}$ and $p_{g}$, go back to step 3 ; Otherwise, continue to Chaos iteration.

Step 6: Determine whether the maximum number of iterations is got, if yes, output the minimum total position $C$, Otherwise, go back to step 3.

\section{TEST AND RESUlt ANALYSIS}

In the course of the experiment, the experimental conditions are as shown in table 1, the node used is shown in Figure 3. The experimental data sets are the RSSI data measured in different communication environments. As shown in Figure 4 deployment, in the indoor hall and outdoor open environment in the $3.2 \mathrm{~m} * 3.2 \mathrm{~m}$ range, according to the location of the unknown node in the environment corresponding to the different communication distance interval,

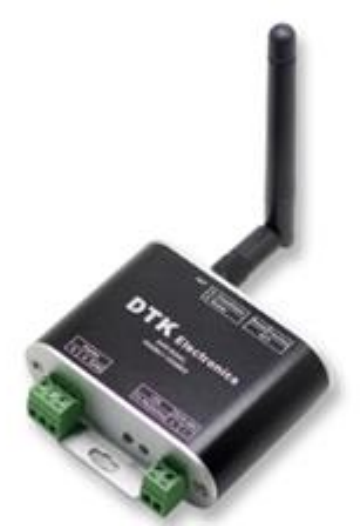

Fig.3 Zigbee module

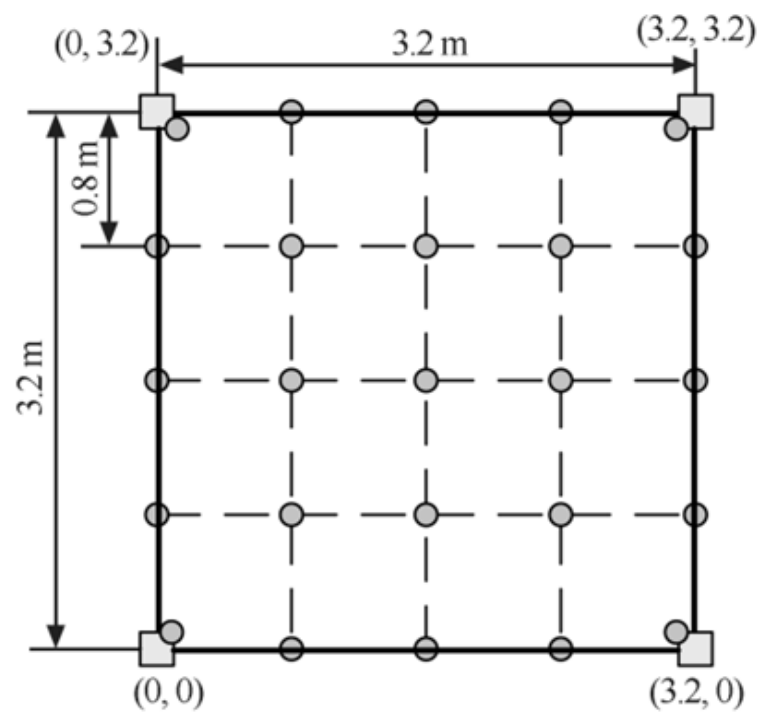

Fig.4 The localization exerimental field

The RSSI values of the unknown node and 4 anchor nodes are measured respectively. 200 sets of RSSI data values are measured at each distance, then the statistical information of RSSI data is computed, and the distribution of interval numbers is expressed. As shown in Figure 4, deploying 4 anchor nodes and one unknown node. By using the current common statistical mean least squares curve fitting method (LSCF), the stepwise regression curve fitting (SRCF), the standard PSO algorithm and the CPSO algorithm proposed to estimate the distance. The RSSI values of the communication between different location points and different anchor nodes in 2 typical environments are estimated respectively, and the absolute error of the experimental results is shown in table 1 and Figure 5. It can be seen from table 1 that the distance estimation precision of PSO algorithm and CPSO algorithm is significantly higher than that of LSCF and SRCF algorithm. Furthermore, the precision of CPSO algorithm is $2.86 \%$ and $5.21 \%$ compared with the accuracy of PSO algorithm in indoor and outdoor respectively.

The outdoor open environment has the least influence on the distance estimation, while the indoor corridor and the hall environment have a great negative impact. This is because indoor corridor and hall communication environment is complex, there are a lot of reflection, diffraction and multipath propagation in communication. and outside the open environment, these conditions are much smaller. Compared with the curve fitting method, the PSO and CPSO methods have smaller estimation errors on the distance estimation method.

Table 1 The distance estimation absolute error of four different methods in two different environments

\begin{tabular}{|l|l|l|}
\hline & Indoor $/ \mathrm{m}$ & Outside $/ \mathrm{m}$ \\
\hline LSCF & 1.1023 & 0.3235 \\
\hline SRCF & 0.9823 & 0.2138 \\
\hline PSO & 0.3319 & 0.0825 \\
\hline CPSO & 0.3224 & 0.0782 \\
\hline
\end{tabular}

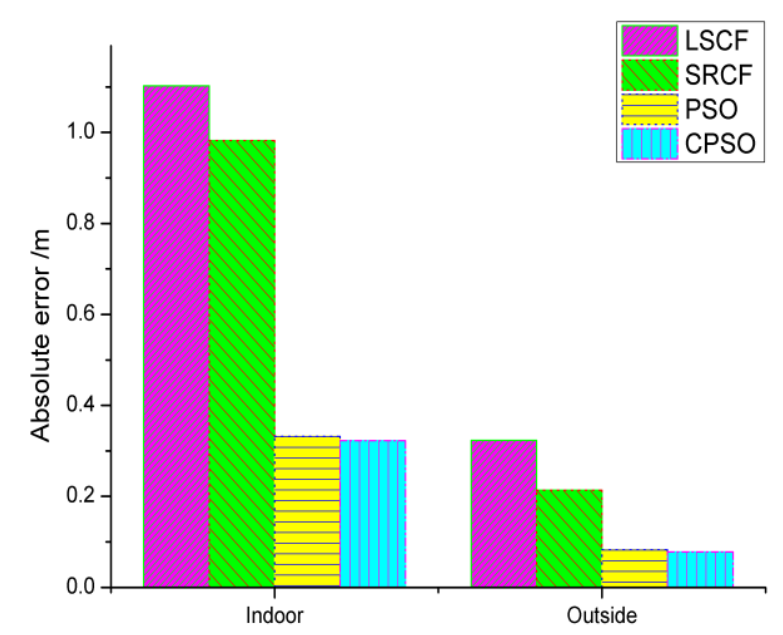

Fig.5 The distance estimation absolute error of four different methods in two different environments 
A. Comparison of the Relationship between Ranging Error and Positioning Accuracy

Aiming at the problem that the range of RSSI algorithm can be affected by environment, it is shown from figure 6 that with the increase of ranging error, the positioning error will increase, but the CPSO algorithm is more reasonable than the existing PSO algorithm, which reduces the effect of the ranging error, and the location error of CPSO algorithm is obviously less affected in the same range error. Especially when the ranging error is large, the improved algorithm has better anti error performance. When position error is 0.9 , the relative range error gap between the PSO and CPSO can reach 5\%, and the trend of this gap will continue to increase. It can be concluded that the CPSO algorithm effectively solves the limitation of the traditional PSO algorithm for ranging correction, and maintains a good precision when the range error is large.

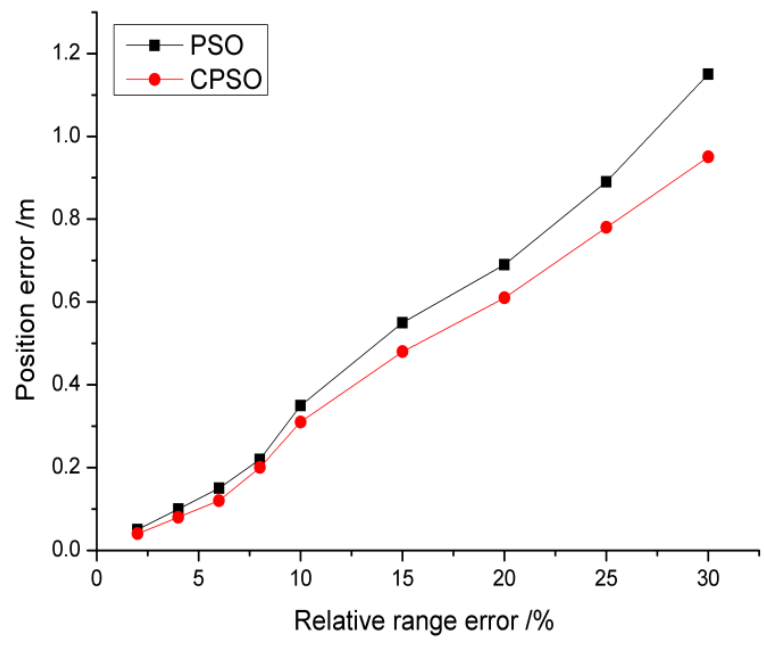

Fig.6 Effect of ranging error on positioning error

\section{B. Error analysis under different communication radius}

The increase of communication radius will reduce the positioning error of nodes to a certain extent, but the excessive communication radius will consume the energy of nodes. In order to compare the average positioning error of each algorithm node under different node communication radius, the proportion of anchor nodes is set to be $20 \%$. The improved algorithm and each localization algorithm are randomly simulated for 100 times to obtain the positioning error.

The test area range is $10 \mathrm{~m} \times 10 \mathrm{~m}$ to $50 \mathrm{~m} \times 50 \mathrm{~m}$, the interval is arranged 25 mobile nodes, 7 reference points, and the location error of 2 algorithms under different communication radius $r$ is shown in Figure 7.

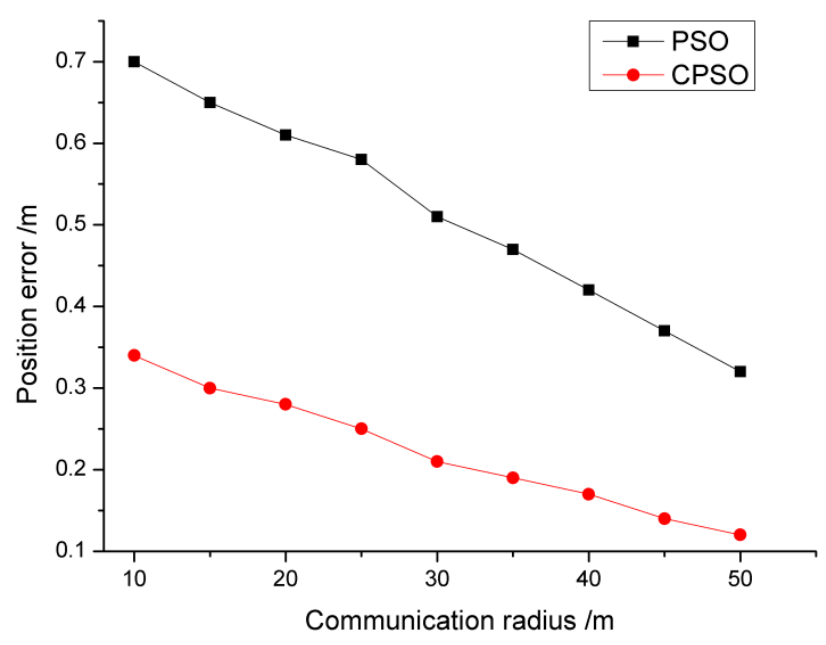

Fig.7 Comparison of location error under different communication radius

It can be seen from Figure 7 that the positioning error of CPSO algorithm decreases with the increase of communication radius of nodes, and the positioning error is smaller than that of PSO. When the node communication radius is $50 \mathrm{~m}$, the positioning error of each localization algorithm is the lowest, and the CPSO positioning error is 0.12 . At the same time, the average error ratio of the existing PSO algorithm is larger than that of the improved CPSO network algorithm, the mininum error of the two algorithms is $0.2 \mathrm{~m}$, the maximum error of the two algorithms is $0.36 \mathrm{~m}$, and average error of the two algorithms is 0.292 . With the increase of radius value, the error of the existing PSO algorithm becomes smaller, but the error of CPSO algorithm decreases with the increase of communication radius.

PSO algorithm has the advantages of parallel operation, less adjustable parameters, fast optimization speed and easy implementation. The disadvantage is that it is easy to fall into the local minimum and the search accuracy is not high. Chaos Particle Swarm Optimization (CPSO) algorithm combines the fast convergence of PSO algorithm and the ergodic randomness of $\mathrm{CO}$ algorithm. In the area near the optimal solution selected by each generation of PSO algorithm, chaos algorithm is used to further search to prevent it from falling into the local optimal value, thus improving the shortcomings of PSO algorithm and becoming an efficient optimization algorithm.

However, the algorithm proposed in this paper has some limitations in correcting the estimated distance between the unknown node and the anchor node. In addition, most of the work in this paper is based on simulation experiments, so there is no more consideration for the complexity of the actual environment.

The improved bat algorithm can be used as an alternative method in this study. Although bat algorithm is superior to other particle swarm optimization algorithms in solving unconstrained optimization problems, it also has defects of other intelligent optimization algorithms. Therefore, we can use cubic mapping to homogenize the speed and position of bat 
population to improve the quality of population data solution, and then introduce Levy flight feature to enhance the ability of the algorithm to jump out of local optimization. After getting the optimal bat value, Powell local search is carried out to accelerate the convergence of the algorithm.

For CPSO algorithm, how to choose more suitable data structure and algorithm parameters, and then improve the running speed of the algorithm is a key problem to be solved in the future. Introducing chaos theory, such as nonlinear dynamics, into the analysis and improvement of particle swarm optimization algorithm is a direction of future development.

\section{CONCLUSION}

At present, the method of line or curve fitting is widely used to estimate the communication distance in wireless sensor network location, which makes the distance estimation error larger, which leads to higher positioning error. In this paper, based on the Rssi location problem in the real WSN system, the statistical distribution characteristics of the measured RSSI data are fully utilized. The improved chaotic particle swarm optimization algorithm is used to estimate the communication distance so as to achieve high precision positioning. In this paper, the CPSO network is used to optimize the RSSI value, then the RSSI value is processed, and the corresponding distance data is obtained. That is to use CPSO training to form a new algorithm, which can avoid local extreme value. The experimental results show that the CPSO has the advantage of local search and global search optimal solution relative to statistical mean least squares fitting, stepwise regression and traditional particle swarm optimization, which increases the convergence rate and global convergence of the algorithm, and improves the positioning accuracy of the rssi-d distance estimation method.

Wireless sensor network location research still have a lot of problems to be solved and perfect, the existing algorithm in the security and privacy issues are relatively fragile, with the development of positioning technology, human life needs, wireless sensor networks in energy consumption, cost, practicality, security positioning, At any time, the research work of the node localization and so on will be the key task of the next stage.

\section{REFERENCES}

[1] Yick, J.; Mukherjee, B.; Ghosal, D. "Wireless sensor network survey",. Computer Networks.vol.52, no.12, pp.2292-2330, 2008.

[2] Halder, S.; Ghosal, A. "A survey on mobility-assisted localization techniques in wireless sensor networks", Journal of Network and Computer Applications. Vol.60, pp.82-94, 2016.

[3] Noppadol Pringsakul, Deacha Puangdownreong, Chaiyo Thammarat, Sarot Hlangnamthip, Obtaining Optimal PIDA Controller for Temperature Control of Electric Furnace System via Flower Pollination Algorithm, WSEAS Transactions on Systems and Control, Volume 14, 2019

[4] Georgi Enchev, Nikolay Djagarov, Zhivko Grozdev, Selecting Type of Communication for Wireless Sensor Network on Board of a Vessel, WSEAS Transactions on Systems and Control, Volume 13, 2018.

[5] Blumrosen G, Hod B, Anker T, et al. "Enhancing RSSI-based tracking accuracy in wireless sensor networks", Acm Transactions on Sensor Networks,vol.9, no.3, pp.1-28, 2013.
[6] Bhuvaneswari, P.; Vaidehi, V.; Saranya, M.A. "Distance based transmission power control scheme for indoor wireless sensor network". In Transactions on Computational Science; Berlin, 2010, pp. 207-222.

[7] Rossi M, Brunelli D, Adami A, et al. "Gas-Drone: Portable gas sensing system on UAVs for gas leakage localization". In Proceedings of the IEEE Sensors , , Valencia, 2014, pp.1431-1434.

[8] Lo G, Gonzalezvalenzuela S, Leung V C. "Wireless body area network node localization using small-scale spatial information", IEEE Journal of Biomedical \& Health Informatics, vol.17, no.3, pp.715-726, 2013.

[9] Zhao J, Xi W, He Y, et al. "Localization of wireless sensor networks in the wild:pursuit of ranging quality", IEEE/ACM Transactions on Networking, vol.21, no.1, pp.311-323, 2013.

[10] Karim, L.; Nasser, N.; Mahmoud, Q.H.; Anpalagan, A.; Salti, T.E. Range-free localization approach for M2M communication system using mobile anchor nodes. J. Netw. Comput. Appl. 2015, 47, 137-146. [CrossRef]

[11] Zhang, T.; He, J.; Zhang, Y. Secure sensor localization in wireless sensor networks based on neural network. Int. J. Comput. Intell. Syst. 2012, 5, 914-923. [CrossRef]

[12] Xie, H.; Li, W.; Li, S.; Xu, B. “An improved DV-Hop localization algorithm based on RSSI auxiliary ranging", In Proceedings of the 2016 35th Chinese Control Conference (CCC), Chengdu, 2016, pp. 8319-8324.

[13] Gui L, Val T, Wei A, et al. "Improvement of range-free localization technology by a novel DV-hop protocol in wireless sensor networks", $A d$ Hoc Networks, vol.24, pp.55-73, 2015.

[14] Dil, B.; Dulman, S.; Havinga, P. "Range-based localization in mobile sensor networks", In Wireless Sensor Networks, Berlin, 2006, pp.164-179.

[15] SAVARESE C, RABAEY J, LANGENDOEN K. "Robust positioning algorithms for distributed ad - hoc wireless sensor network", In Proceedings of the USENIX Technical Annual Conference. Monterey, 2002, pp.317-327.

[16] ZOU Gang, SHI Zhangsong, LIU Zhong. "Comparative study of passive tracking algorithm for distributed particle filter in sensor networks", Chinese Journal of Sensors and Actuators, vol.20, no.6, pp. 1344-1348, 2007.

[17] Years I R. "Amorphous Localization Algorithm Based on BP Artificial Neural Network",. International Journal of Distributed Sensor Networks, vol.22, no.11, pp.6-12, 2015.

[18] Li Z, Liu X, Duan X, et al. "Comparative Research on Particle Swarm Optimization and Genetic Algorithm", Computer \& Information Science, vol.3, no.1, pp.120-127, 2010.

[19] Jordehi A R, Jasni J. "Parameter selection in particle swarm optimisation: a survey", Journal of Experimental \& Theoretical Artificial Intelligence, vol.25, no.4, pp.527-542, 2013.

[20] Kulkarni R V, Venayagamoorthy G K. "Particle Swarm Optimization in Wireless-Sensor Networks: A Brief Survey", IEEE Transactions on Systems Man \& Cybernetics Part C, vol.41, no.2, pp.262-267, 2011.

[21] Wang X, Xu J. "Research on node localization based on praticle swarm optimization for WSNs", Journal of Computer Applications, vol.29, no.2, pp. 494-499, 2009.

[22] Lin C M, Mon Y J, Lee C H, et al. "ANFIS-based Indoor Location Awareness System for the Position Monitoring of Patients", Acta Polytechnica Hungarica, vol.11, no.1, pp.37-48, 2014.

[23] Velimirovic A S, Djordjevic G L, Velimirovic M M, et al. "Fuzzy ring-overlapping range-free (FRORF) localization method for wireless sensor networks" Computer Communications, vol.35, no.13, pp.1590-1600, 2012.

[24] Kulkarni R V, Venayagamoorthy G K. "Bio-inspired Algorithms for Autonomous Deployment and Localization of Sensor Nodes", IEEE Transactions on Systems Man \& Cybernetics Part C, vol.40, no.6, pp.663-675, 2010.

\section{Creative Commons Attribution License 4.0 (Attribution 4.0 International, CC BY 4.0)}

This article is published under the terms of the Creative Commons Attribution License 4.0

https://creativecommons.org/licenses/by/4.0/deed.en_US 